\title{
Myocardial Fibrosis in Hypertrophic Cardiomyopathy: What Remains to be Proven?
}

\author{
Leonardo Sara ${ }^{\circledR}$ \\ CDI - Centro de Diagnóstico por Imagem, Goiânia, GO - Brazil \\ Short Editorial related to the article: Role of Myocardial Fibrosis in Hypertrophic Cardiomyopathy: A Systematic Review and Updated Meta- \\ Analysis of Risk Markers for Sudden Death
}

Hypertrophic cardiomyopathy (HCM) is a complex and well-characterized disease. Its prevalence in the general population is 1 in 500 individuals, with some data suggesting an even higher prevalence when using associated genetic criteria. ${ }^{1}$ This means that, based on a conservative estimate, cities such as Goiânia-GO or Recife-PE have approximately more than 3,000 individuals with the disease, a figure that can be as high as 25,000 individuals in a large city such as São Paulo.

Although HCM is perceived as a rare and usually very severe disease, related to dramatic episodes of sudden death (SD) in young individuals and athletes, it is a disease with low mortality. Due to advances in the diagnosis, treatment and prevention of SD, current estimates indicate a mortality rate of $0.5 \%$ a year, similar to that found in the general population, with most patients with the disease being asymptomatic or minimally symptomatic. ${ }^{2}$ In this scenario, the correct identification of subgroups of individuals with a higher risk of unfavorable outcome and, therefore, a greater chance of benefit when submitted to specific therapeutic strategies, such as the use of implantable cardioverter defibrillators (ICD), is of utmost importance.

However, $\mathrm{HCM}$ is an extremely challenging disease, with variable clinical presentations and an often unpredictable natural history. SD, its most devastating clinical manifestation, occurs most often in previously healthy individuals and unaccompanied by preceding symptoms. Several clinical markers derived from observational studies were organized into risk stratification models, giving origin to the algorithms adopted by the American and European Cardiology Societies. ${ }^{3,4}$

However, the use of such tools has several limitations. The scarcity of randomized studies makes recommendations be based primarily on registries, retrospective or prospective small-scale studies. The accuracy of these models for SD prediction according to validation analyses was moderate (area under the ROC curve ranging from 0.60 to 0.69 ), with low specificity and positive predictive value and sub-optimal performance when used at the individual level.

\section{Keywords}

Cardiomyopathy, Hypertrophic; Prevalence; Death, Sudden, Cardiac/prevention \& control; Heart Failure; Magnetic Resonance Spectroscopy/methods.

\section{Mailing Address: Leonardo Sara •}

CDI - Centro de Diagnóstico por Imagem - Avenida Portugal, 1155, Setor Marista. Postal Code 74150-030, Goiania, GO - Brazil E-mail: leosara@hotmail.com

DOI: $10.5935 / a b c .20190043$
Moreover, individual traditional risk markers have low sensitivity and specificity, and their absence does not safely rule out the chance of SD. ${ }^{5}$ Therefore, the current risk stratification tools in $\mathrm{HCM}$ are imperfect and imprecise, making research on new risk markers a crucial matter.

Cardiac magnetic resonance (CMR) imaging allows the precise identification of areas of myocardial replacement fibrosis through the late enhancement technique. ${ }^{6,7}$ Analyses of prevalence and the distribution pattern of late enhancement in HCM have been widely described. There is a large body of evidence showing that the arrhythmogenic mechanism that would lead to SD in HCM patients is directly related to myocardial fibrosis and, similarly, several studies in patients with $\mathrm{HCM}$ evaluated by magnetic resonance showed a worse prognosis in those with late enhancement such as arrhthmias, heart failure, SD, cardiac death and death from any cause. ${ }^{8}$

In the present article of this issue, ${ }^{9}$ the authors presented a systematic review and meta-analysis that included 21 studies evaluating the association of risk markers with the occurrence of SD. The authors demonstrated a strong correlation between the presence of myocardial fibrosis detected by CMR and the occurrence of arrhythmic outcomes, with a clearly higher relative risk when compared to the other identified risk markers (RR: $3.43 ; 95 \% \mathrm{Cl}$ : 1.95-6.03). This result is in accordance with another meta-analysis evaluating HCM and myocardial fibrosis, totaling 1,063 patients (mean follow-up of $43 \pm 14$ months), in which the presence of late enhancement in these patients resulted in a 9-fold higher chance of ventricular fibrillation / tachycardia and a 3.3-fold higher chance of SD. ${ }^{10}$ These results suggest that late enhancement may be very useful as a high-risk prognostic marker.

About this subject, it should be noted that some considerations are important. Myocardial fibrosis detected by $\mathrm{CMR}$ is very common in $\mathrm{HCM}$ and is observed in approximately $2 / 3$ of the patients. ${ }^{8}$ Therefore, its presence alone does not allow adequate selection of patients candidates for ICD.

Recently, studies have been published establishing a positive and linear association between late enhancement extent and worse prognosis, with the presence of more than $15 \%$ of myocardial fibrosis mass, giving a roughly 2 -fold higher chance of SD when compared to individuals without late enhancement, regardless of other risk markers. ${ }^{11,12}$ However, this approach also has significant limitations. First, there is no universal standardization of the late enhancement quantification technique, leading to the great heterogeneity of results (currently, the most often used techniques are the 6 standard deviation method, the full-with half maximum technique and the Rayleigh Curve Method). ${ }^{8}$ 


\section{Short Editorial}

Moreover, there is still some controversy over whether late enhancement provides clinically relevant incremental information to traditional risk factors, mostly because of the small number of primary outcomes observed in the studies. Additionally, there are no large-scale prospective studies evaluating the late enhancement quantification incorporated into the ACC/AHA and European Cardiology Society algorithms for the prediction of SD in HCM.

$\mathrm{CMR}$ is an extremely valuable tool in the evaluation of $\mathrm{HCM}$ and its application for diagnostic purposes has been very well established. Similarly, the evaluation of myocardial fibrosis by

\section{References}

1. Maron BJ. Clinical Course and Management of Hypertrophic Cardiomyopathy. N Engl J Med. 2018;379(7):655-68.

2. Maron BJ, Maron MS, Rowin EJ. Perspectives on the Overall Risks of Living with Hypertrophic Cardiomyopathy. Circulation. 2017;135:2317-9.

3. Gersh BJ, Maron BJ, Bonow RO, Dearani JA, Fifer MA, Link MS, et al. 2011 ACCF/AHA guideline for the diagnosis and treatment of hypertrophic cardiomyopathy: A report of the American College of Cardiology Foundation/ American Heart Association Task Force on Practice Guidelines Developed in Collaboration with the American Ass. J Am Coll Cardiol. 2011;58(25):212-60.

4. Zamorano JL, Anastasakis A, Borger MA, Borggrefe M, Cecchi F, Charron P, et al. 2014 ESC guidelines on diagnosis and management of hypertrophic cardiomyopathy: The task force for the diagnosis and management of hypertrophic cardiomyopathy of the European Society of Cardiology (ESC). Vol. 35, European Heart Journal. 2014. p. 2733-79.

5. Sen-Chowdhry S, Jacoby D, Moon JC, McKenna WJ. Update on hypertrophic cardiomyopathy and a guide to the guidelines. Nat Rev Cardiol. 2016;13(11):651-75.

6. Moon JCC, McKenna WJ, McCrohon JA, Elliott PM, Smith GC, Pennell DJ. Toward clinical risk assessment in hypertrophic cardiomyopathy with gadolinium cardiovascular magnetic resonance. J Am Coll Cardiol. 2003;41(9):1561-7. the late enhancement technique is a strong prognostic marker of the disease and will probably have a great impact as a risk stratification tool.

Nevertheless, according to the main guidelines on HCM, currently the clinical use of the technique for primary prophylaxis of SD is restricted to ambiguous cases, when the ICD indication is uncertain when using the traditional algorithms..$^{3,4}$ Therefore, to take one step further and for myocardial fibrosis screening to be formally incorporated into the risk stratification routine in HCM, some gaps still need to be addressed.

I think it is a matter of time!
7. Choudhury L, Mahrholdt H, Wagner A, Choi KM, ElliottMD, Klocke FJ, et al. Myocardial scarring in asymptomatic or mildly symptomatic patients with hypertrophic cardiomyopathy. J Am Coll Cardiol.2002;40(12):2156-64.

8. Quarta G, Aquaro GD, Pedrotti P, Pontone G, Dellegrottaglie S, lacovoni $A$, et al. Cardiovascular magnetic resonance imaging in hypertrophic cardiomyopathy: The importance of clinical context. Eur Heart J Cardiovasc Imaging. 2018;19:601-10.

9. Bittencourt MI, Cader SA, Araújo DV, Salles ALF, Albuquerque FN, Spineti PPM, et al. Papel da fibrose miocárdica na cardiomiopatia hipertrófica: Revisão sistemática e metanálise atualizada de marcadores de risco para morte súbita. Arq Bras Cardiol. 2019;112(3):281-289.

10. Reddy ST, Tito A, Filho P, Silva NJ, Doyle M, Yamrozik J, et al. A Systematic review for sudden cardiac death in hyoetrophic cardiomyopathy patients with myocardial fibrosi: a CMR LGE Study. J Am Coll Cardiol. 2012;59(13):E1228.

11. Chan RH, Maron BJ, Olivotto I, Pencina MJ, Assenza GE, Haas T, et al. Prognostic value of quantitative contrast-enhanced cardiovascular magnetic resonance for the evaluation of sudden death risk in patients with hypertrophic cardiomyopathy. Circulation. 2014;130(6):484-95.

12. Weng Z, Yao J, Chan RH, He J, Yang X, Zhou Y, et al. Prognostic Value of LGE-CMR in HCM: A Meta-Analysis. JACC Cardiovasc Imaging. 2016;9(12):1392-402. 